\title{
The Effect of Concomitant Cardiac Resynchronization Therapy on Quality of Life in Patients with Heart Failure Undergoing Cardiac Surgery
}

\author{
Emma Thorén*,1, Milos Kesek ${ }^{2}$, and Lena Jidéus ${ }^{1}$ \\ ${ }^{I}$ Department of Cardiothoracic Surgery, Institution of Surgical Sciences, Uppsala, Sweden \\ ${ }^{2}$ Department of Cardiology, Institution of Medical Sciences, Umeå, Sweden
}

\begin{abstract}
Objectives: To evaluate the effect of concomitant cardiac resynchronization therapy (CRT) on health related quality of life (QoL) in patients with heart failure (HF) and ventricular dyssynchrony undergoing cardiac surgery.

Methods: Twenty-eight patients received permanent epicardial CRT in connection to coronary artery bypass grafting (CABG) and/or aortic valve replacement (AVR) (CRT group). Thirty-seven HF patients without concomitant CRT served as a comparison group (non-CRT group). SF-36 was used to assess QoL in the two groups and was also compared with the general Swedish population.

Results: The median follow-up time was 28 months after surgery (range 8 to 44 months). No difference in QoL could be shown between the CRT group and the comparison group. Several subscales of QoL in the CRT group were in range with the general Swedish population.

Conclusion: Concomitant CRT for patients with HF and ventricular dyssynchrony undergoing CABG and/or AVR did not result in a higher estimated QoL compared to HF patients without CRT.
\end{abstract}

Keywords: Aortic valve replacemen, cardiac resynchronization therapy, coronary artery bypass grafting, heart failure, ventricular dyssynchrony, quality of life.

\section{INTRODUCTION}

Cardiac resynchronization therapy (CRT) is an effective treatment for patients with heart failure (HF) and ventricular dyssynchrony who are symptomatic despite optimal medical treatment $[1,2]$. In selected groups of HF patients, CRT reduces complications and mortality [3-5], decreases clinical symptoms and improves quality of life (QoL) [5-9]. A number of patients undergoing coronary artery bypass grafting (CABG) and/or aortic valve replacement (AVR) surgery suffer from HF and ventricular dyssynchrony, and are consequently considered as candidates for a CRT system. The surgical setting enables epicardial pacing lead placement. The surgical technique results in more successful left lead positioning, same level of clinical improvement, lower left ventricular $(\mathrm{LV})$ related complication rates and lower chronic threshold-capture compared to transvenous lead placement via the coronary sinus [10,11]. Perioperative CRTimplantation in CABG patients seems to carry a positive effect on functional status and QoL in addition to the outcome of surgery $[12,13]$. The information available on concomitant CRT-implantation in association with cardiac surgery is, however, limited. The objective of this study was to evaluate health related QoL in patients with $\mathrm{HF}$ and

*Address correspondence to this author at the Department of Cardiothoracic Surgery, Institution of Surgical Sciences, Uppsala University Hospital, S75185 Uppsala, Sweden; Tel: +46 18611 4016; Fax: +46 18611 3926;

E-mail: emma.thoren@akademiska.se ventricular dyssynchrony receiving a permanent epicardial CRT system during CABG and/or AVR.

\section{MATERIAL AND METHODOLOGY}

This study included patients who underwent CABG, AVR or a combined surgical procedure at the University Hospital of Uppsala, Sweden, between December 2004 and December 2007, meeting the standard criteria for CRT treatment at the time of surgery (sinus rhythm, HF with New York Heart Association (NYHA) Class III-IV, left ventricular ejection fraction (LVEF) $\leq 35 \%$ and QRS duration $\geq 120$ ms or mechanical dyssynchrony on Doppler echocardiography). They were compared to a matched population of patients with reduced LV function $(\mathrm{LVEF}<45 \%)$ undergoing cardiac surgery at the same time. Isolated $\mathrm{CABG}$ patients were matched with two controls while patients who underwent AVR surgery or combined procedures were matched with one control each, due to the scarcity of appropriate controls. Based on preoperative echocardiography, LVEF was classified as "reduced" ( $31 \%$ to $45 \%)$, "severely reduced" $(15 \%$ to $30 \%)$ or "poor" $(<15 \%)$. Subdivision into NYHA class IIIA and IIIB was used, with class IIIA including patients who experienced slight discomfort during normal activities, but able to walk a mile on a flat surface at their own speed and climb stairs slowly without undue discomfort [14]. The study complies with the Declaration of Helsinki. 


\section{Quality of Life Assessment}

The SF-36 is a self-administration questionnaire designed for assessment of health related QoL [15]. It consists of 36 items divided into 8 subscales of health that can be summarized into two main health dimensions: Physical Health Score (PCS) and Mental Health Score (MCS) [16]. The SF36 has been used in a wide range of studies, and normative data on QoL in the general Swedish population have been published [17].

\section{Surgical Techniques}

All patients underwent CABG and/or AVR surgery according to the clinic's routines. The AVR patients received either biological or mechanical valve prosthesis. The CRT group received three permanent epicardial pacing leads and a CRT device during the procedure. The atrial lead was placed on the right atrial wall, between the sinus node and the appendage. The right ventricular lead was implanted on the inferior wall of the right ventricle and the LV lead on the lateral wall of the left ventricle. All three leads were connected to a CRT device placed subcutaneously below the left clavicle and activated during surgery. We used Medtronic CapSure Epi ${ }^{\circledR}$ unipolar and bipolar epicardial leads (4965 and 4968, respectively). The CRT device was Medtronic InSync III ${ }^{\circledR}$ CRT-P in 21 patients, St Jude Medical Frontier II $^{\circledR}$ CRT-P in three patients and St Jude Medical Atlas ${ }^{\circledR}$ CRT-D in one patient. One patient with a previously implanted dual-chamber pacemaker due to a total atrioventricular block received an epicardial LV lead during surgery and the original pacemaker was replaced with a CRT device. In the last two patients, the transvenous leads St Jude Medical Tendril ${ }^{\circledR}$ 1788T, 1636T, 1688T and Riata ${ }^{\circledR}$ Defibril-

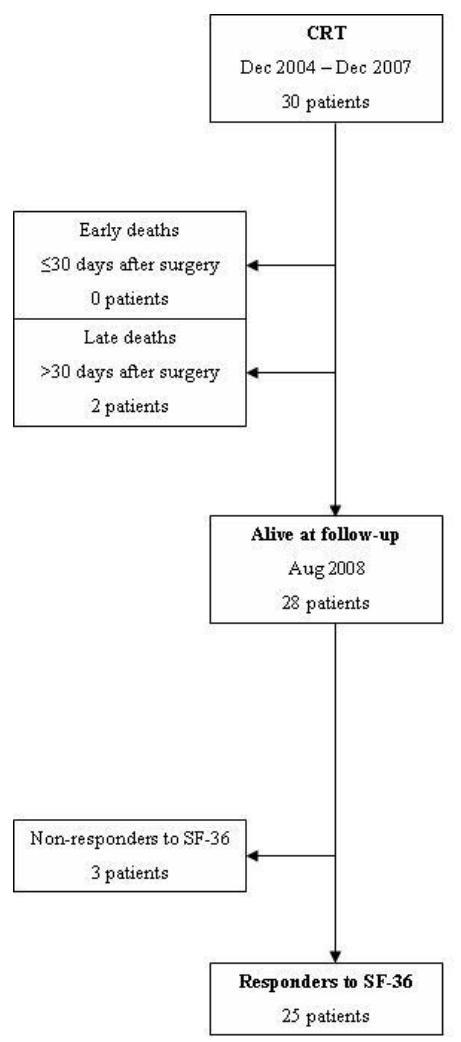

lation Lead 1571 were used in addition to the epicardial LV lead.

\section{Follow-up}

Technical parameters were supervised during routine visits at pacemaker policlinics. At follow-up, the SF-36 (Swedish standard version) was sent to the CRT group and the comparison group. Non-responders received a reminder after four weeks. Incomplete forms were followed up with a telephone call, when possible.

\section{Statistical Analysis}

Statistical analyses were performed with Statistica 8.0 (StatSoft, Inc, Tulsa, OK, USA). Data are presented as mean \pm SD, unless otherwise stated. The Mann-Whitney U test was used for comparison between the groups. $\mathrm{P}$ values $<0.05$ were considered statistically significant.

\section{RESULTS}

Thirty patients received a CRT system. Twenty-eight patients were alive at the time of follow-up (median 27 months; range 8 to 44). During the same period, 78 patients with reduced LV function (LVEF <45\%) without indication for CRT underwent cardiac surgery; 61 of these patients being alive at follow-up (median 32 months; range 10 to 43). The surviving patients were matched for age, main diagnosis and type of surgical procedure to the surviving patients in the CRT group, resulting in 37 patients appropriate for comparison. Patient flow is described in Fig. (1). Baseline patient characteristics are presented in Table $\mathbf{1}$. The technical pa-

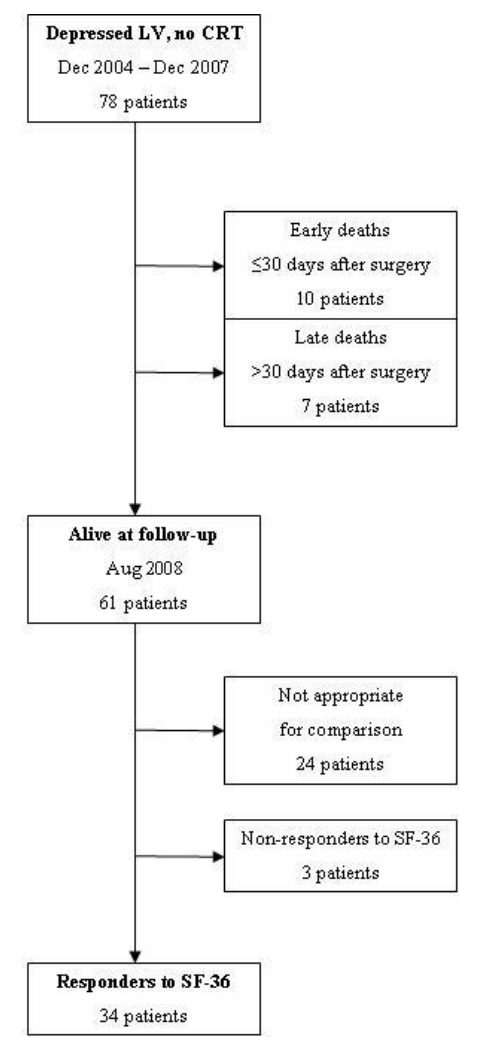

Fig. (1). Patient flow. 
Table 1. Characteristics and Surgical Data of Responder to SF-36

\begin{tabular}{|c|c|c|c|}
\hline \multirow{2}{*}{ Variable } & CRT Group & Comparison Group & \multirow{2}{*}{$p$} \\
\hline & $(\mathbf{n}=\mathbf{2 5})$ & $(\mathrm{n}=\mathbf{3 4})$ & \\
\hline Age (y), median (range) & $72(55-86)$ & $70(46-86)$ & 0.20 \\
\hline Male sex & $22(88)$ & $29(85)$ & $>0.3$ \\
\hline Hypertension & $19(79)$ & $26(76)$ & $>0.3$ \\
\hline Diabetes & $2(8.3)$ & $10(29)$ & 0.03 \\
\hline Ischemic heart disease & $13(54)$ & $23(68)$ & 0.23 \\
\hline S-Creatinine, median (range) & $100(57-165)$ & $97(60-133)$ & $>0.3$ \\
\hline Nt-proBNP, median (range) & $2407(572-27549)$ & $1483(99-16923)$ & 0.12 \\
\hline History of atrial fibrillation & $8(33)$ & $4(8.8)$ & 0.06 \\
\hline LBBB & $10(40)$ & $1(2.9)$ & $<0.001$ \\
\hline QRS width (ms), median (range) & $138(94-200)$ & $107(82-178)$ & $<0.001$ \\
\hline LVEF & & & 0.24 \\
\hline Reduced (31-45\%) & $1(4.2)$ & $5(15)$ & \\
\hline Severely reduced (15-30\%) & $22(88)$ & $27(79)$ & \\
\hline Poor $(<15 \%)$ & $2(8.3)$ & $2(5.9)$ & \\
\hline NYHA class & & & $<0.001$ \\
\hline I-II & $0(0)$ & $6(18)$ & \\
\hline IIIA & $5(20)$ & $16(47)$ & \\
\hline IIIB & $20(80)$ & $12(35)$ & \\
\hline Procedure & & & 0.11 \\
\hline CABG & $8(32)$ & $18(53)$ & \\
\hline $\mathrm{AVR} \pm \mathrm{CABG}$ & $17(68)$ & $16(47)$ & \\
\hline
\end{tabular}

Data shown as numbers (percentages within group) unless otherwise stated. AVR = aortic valve replacement; CABG = coronary artery bypass grafting; CRT = cardiac resynchronization therapy; LBBB = left bundle branch block; LVEF = left ventricular ejection fraction; NYHA class = New York Heart Association functional classification.

rameters of the epicardial leads during the follow-up period were adequate, with median LV lead pacing thresholds $0.5 \mathrm{~V}$ at $0.4 \mathrm{~ms}$. At three and six months after implantation, the average ventricular pacing percentage was $97 \%$ and $99 \%$, respectively. The response rate to the SF-36 was $89 \%$ (25 of 28 patients) in the CRT group and 92\% (34 of 37) in the comparison group.

Two of the thirty CRT patients $(6.7 \%)$ had died at follow-up compared to 17 of $78(22 \%)$ patients with depressed LV function but without CRT, see Fig. (1). There were no early deaths ( $\leq 30$ days after surgery) among the CRT patients and ten early deaths in the comparison group $(p=0.04)$.

\section{Quality of life}

The eight subscales of the SF-36 indicated similar levels of QoL after surgery in the CRT group and the comparison group (Table 2). With the scores summarized into the two main dimensions of health the CRT group showed a similar
Physical Health Score but a higher Mental Health Score (Table 2). Within the CRT group, no difference in QoL could be detected between patients with typical left bundle branch block ( $\mathrm{n}=10$; PCS 46 \pm 11 ; MCS $51 \pm 11)$ and patients

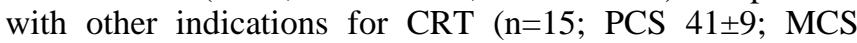
49 \pm 12 ), PCS $p=0.2$; MCS $p=0.6$.

Compared to the general Swedish population the CRT group scored lower in the summarized Physical Health Score, as well as some of the SF-36 subscales considered as predominantly physical (Table 2). The summarized Mental Health Score and scores in the majority of the SF-36 subscales associated with mental health did not differ between the CRT group and the general Swedish population.

\section{DISCUSSION}

The main finding of this study was that in HF patients with ventricular dyssynchrony undergoing $\mathrm{CABG}$ and/or AVR surgery, concomitant CRT did not result in a higher 
Table 2. Quality of Life After Cardiac Surgery with and Without Concomitant CRT, and in a Sample of the General Swedish Population

\begin{tabular}{|l|c|c|c|c|c|}
\hline \multicolumn{1}{|c|}{ SF-36 Variable } & CRT Group (n=25) & Comparison Group (n=34) & $\boldsymbol{p}^{\boldsymbol{a}}$ & Swedish Population & $\boldsymbol{p}^{\boldsymbol{b}}$ \\
\hline \hline Physical functioning & $65.9 \pm 21.8$ & $69.1 \pm 26.3$ & $>0.3$ & $87.9 \pm 19.6$ & $<0.001$ \\
\hline Role limitation, physical & $49.0 \pm 46.5$ & $53.7 \pm 43.6$ & $>0.3$ & $83.2 \pm 31.8$ & $<0.001$ \\
\hline Bodily pain & $80.2 \pm 21.6$ & $79.8 \pm 27.9$ & $>0.3$ & $74.8 \pm 26.1$ & 0.3 \\
\hline General health & $71.0 \pm 17.7$ & $65.3 \pm 22.3$ & $>0.3$ & $75.8 \pm 22.2$ & 0.3 \\
\hline Vitality & $69.0 \pm 19.4$ & $58.7 \pm 25.7$ & 0.1 & $88.8 \pm 22.8$ & $>0.3$ \\
\hline Social functioning & $89.1 \pm 18.2$ & $76.5 \pm 27.8$ & 0.1 & $88.6 \pm 20.3$ & $>0.3$ \\
\hline Role limitation, emotional & $54.7 \pm 47.0$ & $53.5 \pm 44.8$ & $>0.3$ & $85.7 \pm 29.2$ & $<0.001$ \\
\hline Mental health & $83.5 \pm 17.2$ & $73.4 \pm 21.7$ & 0.1 & $80.9 \pm 18.9$ & $>0.3$ \\
\hline Physical health score & $42.9 \pm 9.9$ & $45.7 \pm 11.3$ & 0.3 & $50.0 \pm 9.7$ & $<0.001$ \\
\hline Mental health score & $50.0 \pm 11.5$ & $43.5 \pm 12.2$ & 0.04 & $50.0 \pm 10.3$ & $>0.3$ \\
\hline
\end{tabular}

Data shown as mean $( \pm \mathrm{SD}) .{ }^{\mathrm{a}} \mathrm{SF}-36$ scores in the CRT group vs the comparison group. ${ }^{\mathrm{b}} \mathrm{SF}-36$ scores in the CRT group vs the general Swedish population $(\mathrm{n}=8004 ; 15-80$ years; $48 \%$ male $)$. CRT $=$ cardiac resynchronization therapy.

estimated QoL compared to patients with reduced LV function not eligible for CRT. In contrast, a limited number of other studies have shown a beneficial effect of CRT and epicardial leads placed in association to cardiac surgery. In a small study on CRT with epicardial leads during CABG in patients with HF [12], a better estimated QoL was seen in patients with active CRT. Similarly, CRT improved QoL and LV systolic function after CABG in HF patients with ventricular dyssynchrony [13].

Our CRT group included a higher percentage of patients initially in NYHA-class IIIB (80\%) compared to the comparison group (35\%). It would therefore be reasonable to expect a lower resulting QoL score in the CRT group. We did not detect any adverse effect of CRT on QoL. The implantation and subsequent follow-up of the CRT device were well tolerated and did not significantly affect the scores reflecting the mental and physical well-being of the patients. The difference in the initial condition might have been opposed by a hidden beneficial effect of CRT.

Interestingly, the mortality in the CRT group was lower after surgery than in the matched comparison group. For early deaths this difference was significant. CRT concomitant with CABG has previously been associated with low mortality 18 months after surgery [13]. The beneficial effect of CRT may thus be most apparent early in the postoperative period and loose significance later in the course, as reflected in our QoL measurements more than two years after surgery.

The estimated statistical power to detect a difference in mean PCS (or MCS) of 7 units is $76 \%$ in a reasonable model of present setting (mean PCS values 43 and 50, SD 10, current sample sizes, two-tailed test, probability of alpha error $5 \%$ ). Such an additional effect of CRT on QoL after the operation is therefore improbable. The statistical power to detect a difference of 4 units (mean PCS 43 and 47) decreas- es to $33 \%$. An effect of this smaller magnitude may thus be present, but undetected.

The summarized Mental Health Score was significantly higher in the CRT group and in range with the general Swedish population, assessed nearly four years after surgery. None of the four subscales concerned with mental health, however, differed. The reason is unclear for a higher reported Mental Health Score in the CRT group. A placebo effect might be a possible explanation. As described by Mandeep et $a l$, CRT can exert a substantial placebo effect with improved QoL parameters [18].

Implantation of a permanent CRT system during cardiac surgery is a relatively small additional procedure. We did not detect any negative influence on QoL from CRT. Other authors have reported positive findings $[12,13]$ and CRT may have a role in the treatment in selected patients with HF and ventricular dyssynchrony undergoing CABG and/or AVR surgery.

\section{Limitations}

This was a nonrandomized retrospective study on a relatively small population and the limited number of patients suitable for comparison restricted the matching process. The comparison group did not fulfill the CRT criteria, although the LV function was depressed to a similar degree. This may have affected the comparison results. No baseline data for QoL was available and individual patient differences in QoL were not possible to assess.

\section{CONCLUSION}

Concomitant CRT for HF patients with ventricular dyssynchrony undergoing CABG and/or AVR did not result in a higher estimated QoL compared to HF patients without CRT. Further studies are needed to draw conclusions about 
the possible long-term benefits of CRT in patients undergoing cardiac surgery.

\section{CONFLICT OF INTEREST}

The authors confirm that this article content has no conflicts of interest.

\section{ACKNOWLEDGEMENTS}

Declared none.

\section{REFERENCES}

[1] Dickstein K, Cohen-Solal A, Filippatos G, et al. ESC Guidelines for the diagnosis and treatment of acute and chronic heart failure 2008: the Task Force for the diagnosis and treatment of acute and chronic heart failure 2008 of the European Society of Cardiology. Developed in collaboration with the Heart Failure Association of the ESC (HFA) and endorsed by the European Society of Intensive Care Medicine (ESICM). Eur Heart J 2008; 29: 2388-442.

[2] Vardas PE, Auricchio A, Blanc JJ, et al; European Society of Cardiology; European Heart Rhythm Association. Guidelines for cardiac pacing and cardiac resynchronization therapy: The Task Force for cardiac pacing and cardiac resynchronization therapy of the European Society of Cardiology. Developed in collaboration with the European Heart Rhythm Association. Eur Heart J 2007; 28: 2256-95.

[3] Bradley DJ, Bradley EA, Baughman KL, et al. Cardiac resynchronization and death from progressive heart failure: A meta-analysis of randomized controlled trials. JAMA 2003; 289: 730-40.

[4] Bristow MR, Saxon LA, Boehmer J, et al; Comparison of Medical Therapy, Pacing, and Defibrillation in Heart Failure (COMPANION) Investigators. Cardiac-resynchronization therapy with or without an implantable defibrillator in advanced chronic heart failure. N Engl J Med 2004; 350: 2140-50.

[5] Cleland JG, Daubert JC, Erdmann E, et al; Cardiac Resynchronization-Heart Failure (CARE-HF) Study Investigators. The effect of cardiac resynchronization on morbidity and mortality in heart failure. N Engl J Med 2005; 352: 1539-49.
[6] Abraham WT, Fisher WG, Smith AL, et al; MIRACLE Study Group. Multicenter InSync Randomized Clinical Evaluation. Cardiac resynchronization in chronic heart failure. N Engl J Med 2002; 346: 1845-53.

[7] De Marco T, Wolfel E, Feldman AM, et al. Impact of cardiac resynchronization therapy on exercise performance, functional capacity, and quality of life in systolic heart failure with QRS prolongation: COMPANION trial sub-study. J Cardic Fail 2008; 14: 9-18.

[8] Molhoek SG, Bax JJ, Bleeker GB, et al. Long-term follow-up of cardiac resynchronization therapy in patients with end-stage heart failure. J Cardiovasc Electrophysiol 2005; 16: 701-7.

[9] Linde C, Leclercq C, Rex S, et al. Long-term benefits of biventricular pacing in congestive heart failure: Results from the MUltisite STimulation In Cardiomyopathy (MUSTIC) study. J Am Coll Cardiol 2002; 40: 111-8.

[10] Atoui R, Essebag V, Wu V, et al. Biventricular pacing for endstage heart failure: Early experience in surgical vs. transvenous left ventricular lead placement. Interact Cardiovasc Thorac Surg 2008, 7: 839-44.

[11] Mair H, Sachweh J, Meuris B, et al. Surgical epicardial left ventricular lead versus coronary sinus lead placement in biventricular pacing. Eur J Cardiothorac Surg 2005; 27: 235-42.

[12] Goscinska-Bis K, Bis J, Krejca M, et al. Totally epicardial cardiac resynchronization therapy system implantation in patients with heart failure undergoing CABG. Eur J Heart Fail 2008; 10: 498-506.

[13] Pokushalov E, Romanov A, Prohorova D, et al. Coronary artery bypass grafting with concomitant cardiac resynchronisation therapy in patients with ischaemic heart failure and left ventricular dyssynchrony. Eur J Cardiothorac Surg 2010; 38: 773-80.

[14] Dolgin M, Ed; and the Criteria Committee of the New York Heart Association. Nomenclature and criteria for diagnosis of diseases of the heart and great vessels, 9th ed. Boston: Little Brown \& Co Inc 1994.

[15] Sullivan M, Karlsson J, Taft C, Ware JE. SF-36 Health survey: Swedish manual and interpretation guide. 2nd ed. Gothenburg: Sahlgrenska University Hospital 2002.

[16] Ware JE, Kolinski M, Keller SD. SF-36 Physical and mental health summary scales: A user's manual. Boston, MA: New England Medical Center, the Health Institute 1994.

[17] Sullivan M, Karlsson J. The Swedish SF-36 Health Survey III. Evaluation of criterion-based validity: Results from normative population. J Clin Epidemiol 1998; 51: 1105-13.

[18] Mandeep R, Mehra MR, Greenberg BH. Cardiac resynchronization therapy: Caveat medicus! J Am Coll Cardiol 2004; 43: 1145-8.

Received: November 11, 2013

Revised: January 15, 2014

Accepted: January 20, 2014

(C) Thorén et al.; Licensee Bentham Open.

This is an open access article licensed under the terms of the Creative Commons Attribution Non-Commercial License (http://creativecommons.org/licenses/ by-nc/3.0/) which permits unrestricted, non-commercial use, distribution and reproduction in any medium, provided the work is properly cited. 